\title{
IDENTIFICAÇÃO DE SISTEMAS NÃO LINEARES USANDO MÉTODOS POR SUBESPAÇOS
}

\author{
SANTOS D. M. BORJAS ${ }^{1}$, ClaUdio GARCiA ${ }^{2}$. \\ 1. Depto. DCEN, Universidade Federal Rural do Semi Árido-UFERSA \\ Av. Francisco Mota 570, Bairro Costa e Silva, 59625-900, Mossoró,RN, Brasil \\ E-mails: santos.borjaseufersa.edu.br
}

\author{
2. Laboratório de Automação e Controle, Escola Politécnica, Universidade de São Paulo \\ Av. Prof. Luciano Gualberto, trav. 3, 158, Cidade Universitária, 05508-900, São Paulo, SP, Brasil \\ E-mails: clgarcia@lac.usp.br
}

\begin{abstract}
This paper presents a methodology for the Nonlinear system identification of a Wiener type model, using methods for subspaces and polynomials of Chebyshev. The subspace methods used are MOESP and N4SID. A simulated example is presented to compare the performance of these algorithms.
\end{abstract}

Keywords_- System identification, structure of Wiener, Subspace identification.

Resumo — Neste artigo é apresentada uma metodologia para a identificação de um sistema não linear do tipo Wiener, usando métodos por subespaços e polinômios de Chebyshev. Os métodos por subespaços usados são MOESP e N4SID. Um exemplo simulado é apresentado para comparar o desempenho destes algoritmos.

Palavras-chave— Identificação de sistemas, Estrutura de Wiener, Identificação por subespaços.

\section{Introdução}

A maioria dos processos industriais é não linear, mas a planta pode ser identificada com modelos lineares, se eles forem linearizados em torno de um ponto de operação. No entanto, há sistemas não lineares que têm dinâmica complexa, não sendo possível aproximá-los por sistemas lineares. Um modo de contornar este problema é restringir a operação do processo em certa faixa, em que o sistema tenha comportamento linear. Um caso típico são as válvulas de controle, as quais podem ter um comportamento linear se trabalharem, por exemplo, na faixa de $10 \%$ a $90 \%$ de abertura. No entanto, em muitos casos práticos é preciso que o sistema opere dentro da faixa de saturação, motivo pelo qual o modelo deve refletir esta não linearidade. A necessidade de descrever de forma mais precisa o comportamento não linear de sistemas reais levou à busca de representações não lineares usando métodos de séries funcionais, como as séries de Volterra [Volterra, 1959]. A desvantagem delas é a complexidade computacional, a dificuldade de incorporar informações a priori e de interpretar e estimar características físicas do processo a partir do modelo [Billings, 1980]. Como opção para esses problemas, Billings (1980) propôs os sistemas orientados a blocos, em que se separa o modelo em um subsistema linear dinâmico e um não linear estático. Dependendo da posição dos blocos, o modelo não linear é chamado de sistema de Hammerstein ou de Wiener. Para essas representações, Billings (1980) sugeriu novas pesquisas para simplificar e estender a aplicação de tais métodos. Uma das vantagens de se usar modelos por blocos na identificação de sistemas não lineares é poder usar técnicas de identificação linear já consolidadas, como os métodos por subespaços.

A identificação de sistemas para modelos do tipo Hammerstein ou Wiener vem sendo pesquisada nos últimos anos [Greblicki; Pawlak, 1991], [Zhu, 1999], [Pearson; Pottmann, 2000], [Bloemen; at al, 2001], [Saha; at al, 2004], [Biagiola; Figueroa, 2011]. Recentemente os métodos de identificação por subespaços têm sido propostos para identificar modelos do tipo Wiener e Hammerstein [Westwick; Verhaegen, 1996], [Lovera; at al, 2000], [Goethals; at al, 2004].

Este artigo busca avaliar o desempenho dos métodos MOESP (Multivariable Output-Error State sPace) e N4SID (Numerical algorithms for Subspace Satate Space System Identification), usando os polinômios de Chebyshev, na identificação de um modelo de Wiener para um sistema em malha aberta.

\section{Identificação determinística por subespaços}

Os modelos por subespaços estão relacionados com sistemas e modelos da forma

$$
\begin{aligned}
& x_{k}=A x_{k}+B u_{k} \\
& y_{k}=C x_{k}+D u_{k}
\end{aligned}
$$

onde os vetores $u_{k} \in \mathfrak{R}^{m}$ e $y_{k} \in \mathfrak{R}^{l}$ são, respectivamente os valores medidos das entradas e saídas no instante $k$ dos processos com $m$ entradas e $l$ saídas. O vetor $x_{k} \in \mathfrak{R}^{n}$ é o vetor de estados do processo em tempo discreto no instante $k . A, B$, $C$ e $D$ são matrizes de dimensões apropriadas. 


\subsection{Problema da identificação determinística}

Dado um conjunto de entradas $u_{k}$ e saídas $y_{k}$, determine a ordem $n$ do sistema desconhecido e as matrizes $(A, B, C, D)$ [Van Overschee; De Moor, 1996].

\subsection{Solução ideal}

As Eq. (1) e (2) podem ser expressas na forma matricial

$$
\left\lfloor\begin{array}{c}
\boldsymbol{X}_{k+1} \\
\boldsymbol{Y}_{k}
\end{array}\right\rfloor=\boldsymbol{\theta}\left\lfloor\begin{array}{l}
\boldsymbol{X}_{k} \\
\boldsymbol{U}_{k}
\end{array}\right\rfloor
$$

onde $\boldsymbol{\theta}=\left\lfloor\begin{array}{ll}A & B \\ C & D\end{array}\right]$ corresponde aos parâmetros desconhecidos. A equação (3) pode ser interpretada como um modelo de regressão. Se na equação (3) as matrizes $\boldsymbol{X}_{k+1}, \boldsymbol{Y}_{k}, \boldsymbol{X}_{k}$ e $\boldsymbol{U}_{k}$ são dadas, então o parâmetro desconhecido $\boldsymbol{\theta}$ pode ser calculado pelo método dos mínimos quadrados, isto é:

$$
\hat{\theta}=\left[\begin{array}{cc}
\hat{A} & \hat{B} \\
\hat{C} & \hat{D}
\end{array}\right]=\min _{A, B, C, D}\left\|\left[\begin{array}{c}
X_{k+1} \\
Y_{k}
\end{array}\right]-\theta\left[\begin{array}{l}
X_{k} \\
U_{k}
\end{array}\right]\right\|_{F}^{2}
$$

onde $\hat{\theta}$ denota a estimativa de $\theta$ e \|\|$_{F}^{2}$ denota a norma de Frobenius de uma matriz. Da equação (4), resulta:

$$
\hat{\boldsymbol{\theta}}=\left[\begin{array}{c}
\boldsymbol{X}_{k+1} \\
\boldsymbol{Y}_{k}
\end{array}\right]\left[\begin{array}{l}
\boldsymbol{X}_{k} \\
\boldsymbol{U}_{k}
\end{array}\right]^{\boldsymbol{T}}\left(\left[\begin{array}{l}
\boldsymbol{X}_{k} \\
\boldsymbol{U}_{k}
\end{array}\right]\left[\begin{array}{l}
\boldsymbol{X}_{k} \\
\boldsymbol{U}_{k}
\end{array}\right]^{T}\right)^{-1}
$$

Então, em um caso ideal, quando se tem os dados de entrada, saída e a sequiência de estados para dois instantes de tempo sucessivos $k$ e $k+1$, a identificação do parâmetro $\boldsymbol{\theta}$ na equação (3) é trivial. No entanto, na prática, $\boldsymbol{X}_{k+1}$ e $\boldsymbol{X}_{k}$ não são conhecidos e têm que ser estimados a partir dos dados de entrada e saída. Isto é um ponto importante nos métodos de identificação por subespaços. A diferença entre estes métodos reside na forma de como obter a sequência de estados estimados.

\subsection{Equações matriciais}

Fazendo iterações sucessivas na equação (1)-(2), obtém-se a seguinte equação matricial

$$
Y_{f}=\Gamma_{n} X_{f}+H U_{f}
$$

onde o símbolo $t$ denota dados futuros e $p$ dados passados. $\Gamma_{n}$ é a matriz de observabilidade estendida e é definida por

$$
\Gamma_{n}=\left(C^{T}(C A)^{T} \ldots\left(C A^{n-1}\right)^{T}\right)^{T}
$$

A matriz $H$ é definida como em [Van Overschee; De Moor, 1996]. Os dados passados e futuros das matrizes em blocos de Hankel são definidos por:

$U_{p}=\left\lfloor\begin{array}{ccc}u_{0} & \ldots & u_{j-1} \\ \vdots & \ldots & \vdots \\ u_{i-1} & \ldots & u_{i+j-2}\end{array}\right\rfloor$.

De forma similar são definidas as outras matrizes $U_{f}, Y_{p}, Y_{f}$ e $W_{p}$.

A sequência de estados $\boldsymbol{X}_{k}$ pode ser computada usando projeção ortogonal ou projeção oblíqua, para maiores detalhes ver [Borjas; Garcia, 2012].

\subsection{Método MOESP e método N4SID}

Os métodos de identificação por subespaços mais usados são MOESP e N4SID. O método MOESP soluciona o problema da identificação determinística aplicando certa projeção ortogonal na equação (6), obtendo-se uma aproximação da matriz de observabilidade estendida $\Gamma_{n}$, a partir da qual se computam as matrizes $A$ e $C$. Para calcular as matrizes $B$ e $D$ vide [Verhaegen, 1994].

Para solucionar o problema da identificação determinística, o método N4SID aplica certa projeção oblíqua na equação (6), obtendo-se uma aproximação para a sequência de estados $\boldsymbol{X}_{k+1}$ e $\boldsymbol{X}_{k}$. As matrizes do sistema $(A, B, C, D)$ são aproximadas usando a equação (5). Para maiores detalhes vide [Van Overschee; De Moor, 1996].

\section{Polinômios de Chebyshev}

Muitos pesquisadores usam os polinômios de Chebyshev junto com outra estrutura de modelo para identificar sistemas não lineares, dentre os quais se pode citar [Namatame; Ueda, 1992], [Shingu; at al, 1997], [Prata; Kot, 2002], [Purwar, at al, 2003]. Os polinômios de Chebyshev são definidos por [Rivlin, 1990]

$$
T_{n}(x)=\cos \left(n \cos ^{-1}(x)\right)
$$

para $|x| \leq 1$ e $n \geq 0$. Estes polinômios são ortogonais para $x_{i}=\cos (i 2 \pi / N) \operatorname{com} i=0,1, . ., N-1$. Os polinômios de Chebyshev de primeira ordem são definidos pela relação recursiva:

$$
T_{n+1}(x)=2 x T_{n}(x)-T_{n-1}(x)
$$

Da equação (9) tem-se que $T_{0}(x)=1$ e $T_{1}(x)=x$, portanto $T_{n}(x)$ é um polinômio de grau $n$ com coeficiente dominante $2^{n-1}$, quando $n \geq 1$. 
Seja a função

$$
z_{k}=f\left(y_{k}, n\right)
$$

onde $f$ é uma função não linear definida no intervalo [a,b]. Usando (9), a equação (10) pode ser representada por

$$
z_{k}=c_{0} T_{0}\left(y_{k}\right)+. .+c_{n} T_{n}\left(y_{k}\right)
$$

onde $n$ representa a ordem do polinômio e $c_{i}$ representa as coordenadas da função $f$. No caso que seja conhecido o vetor $T=\left[T_{0}\left(y_{k}\right) \ldots . T_{n}\left(y_{k}\right)\right]$, é possível obter uma estimativa da função não linear, sendo que suas coordenadas são estimadas de

$$
c_{i}=[T]^{+} z_{k}
$$

Na equação (12) o símbolo + representa a Pseudo Inversa de Moore-Penrose.

\section{Modelo de Wiener}

Os modelos por blocos interconectados são estruturas eficientes na modelagem de sistemas não lineares. Uma estrutura de Wiener é composta por um bloco linear dinâmico e um bloco não linear estático, conforme indicado na Figura 1.

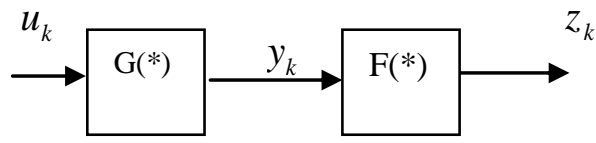

Figura 1. Modelo de Wiener.

onde $u_{k}$ e $z_{k}$ representam os sinais de entrada e saída do sistema, respectivamente. $G(*)$ representa o bloco dinâmico linear e $F(*)$ o bloco estático nãolinear.

O modelo em espaços de estados de um sistema de Wiener é representado pelas equações (1) - (2) e $z_{k}=F\left(y_{k}\right)+v_{k}$. $v_{k}$ é ruído branco com média zero.

\subsection{Problema da identificação não linear}

Dado um conjunto de dados de entradas $u_{k} \mathrm{e}$ saídas $z_{k}$, determine uma estimativa para $G(*)$ e $F(*)$.

\section{Identificação do modelo de Wiener usando métodos por subespaços e polinômios de Chebyshev}

Esta secção apresenta uma metodologia para se obter uma representação do modelo de Wiener a partir dos dados de entrada e saída. A grande dificuldade para se obter a representação do modelo de Wiener está na necessidade de estimar a função $F(*)$ a partir dos dados de entrada e saída, não se tendo disponível o sinal intermediário $y_{k}$. A metodologia consiste dos seguintes passos:

1. Dado um conjunto de entradas $u_{k}$ e saídas $z_{k}$, determine uma estimativa para $G(*)$.

2. Dado o sinal de entrada $u_{k}$ e o sistema linear estimado $G(*)$, determine uma estimativa para $y_{k} \approx \tilde{y}_{k}$.

3. Dado o sinal estimado $\tilde{y}_{k}$ e o sinal de saída $z(k)$, determine uma estimativa para a função não linear $F(*)$.

4. Validar os modelos.

O bloco linear $G(*)$ é estimado usando a identificação por subespaços, descrita na Seção 2. A não linearidade estática $F(*)$ pode ser estimada através de séries de Taylor, ajuste de curvas, aproximação polinomial ou ferramentas de otimização. Visando se ter um erro mínimo de aproximação, são usados neste trabalho os polinômios de Chebyshev descritos na Seção 3. A ordem $n$ dos polinômios de Chebyshev é definida pelo usuário.

\section{Simulações, resultados e discussões}

Nesta seção é apresentada a identificação em malha aberta para um exemplo simulado aplicando a metodologia proposta, tratando-se de um caso SISO com estrutura de Wiener. O detalhamento deste exemplo simulado pode ser encontrado em [Wigren, 1994]. A função de transferência da parte linear do sistema de Wiener é dada por

$G(z)=\frac{0.1578 z+0.1379}{z^{2}-1.375 z+0.6703}$

A função não linear apresenta uma estrutura conhecida como saturação, como mostra a Figura 2 e é definida por

$$
F(y)= \begin{cases}1.5 & y>1.5 \\ y & 0.5 \leq y \leq 1.5 \\ 0.5 & y<0.5\end{cases}
$$

Dada as funções $G(z)$ e $F(y)$, o passo seguinte é coletar os dados de entrada e saída. Estes dados são gerados usando os seguintes comandos do Matlab: $\mathrm{N}=2000$; (número de dados a coletar) ny $=1$; (número de saídas) $a=1 ; b=1 ;(a=$ media e $b=$ desvio padrão $)$ $\mathrm{u}=\mathrm{a}+\mathrm{b} . * \operatorname{randn}(\mathrm{n}, \mathrm{m})$; $\mathrm{y}=\mathrm{dl} \operatorname{sim}(\mathrm{num}, \mathrm{dem}, \mathrm{u})$; $\mathrm{z}=(\mathrm{y}<.5) * .5+((\mathrm{y}>=.5) \&(\mathrm{y}<=1.5)) . * \mathrm{y}+(\mathrm{y}>1.5) * 1.5$; 


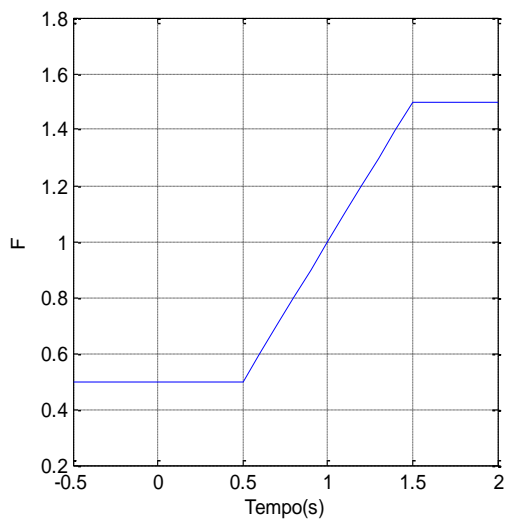

Figura 2. Representação da função não linear $F$.

Foram coletados 2000 pontos de entrada $u$ e saída $y$, dos quais 1500 foram usados na identificação e o restante na validação. Um trecho dos sinais pré-tratados usados na identificação é mostrado na Figura 3. Agora deve-se encontrar um modelo que determine a melhor estimativa para a função $G(*)$. Neste trabalho são usados os modelos MOESP e N4SID, em que MOESP, denota o algoritmo MOESP (caso determinístico) implementado por Michael Verhaegen e N4SID se encontra como Toolbox do Matlab.
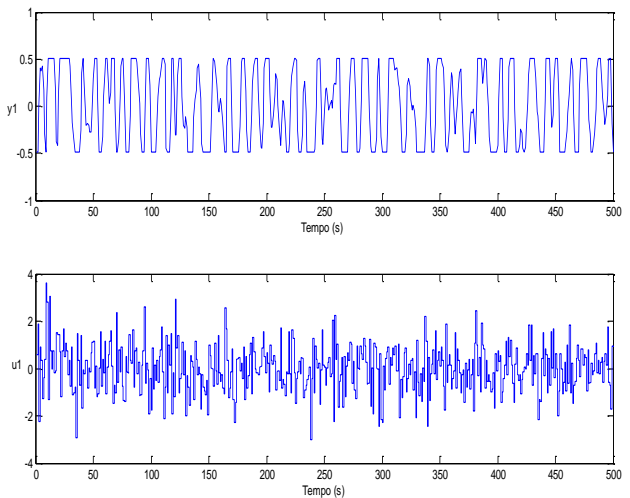

Figura 3. Parte dos dados de entrada $u$ e saída $y$ usados na identificação.

A ordem $n=2$ mostrada na Figura 4 é dada pelos valores mais significativos da matriz $S$, a qual é obtida da SVD (decomposição em valores singulares) de certas projeções oblíquas ou ortogonais, dependendo do algoritmo sendo aplicado [Overschee; De Moor, 1996].

As matrizes do sistema linear obtida pelo método N4SID são dadas pelo seguinte algoritmo:

th $=$ n4sid $([$ ze ue $], 1: 10,1,30,[1,0,0])$

[An,Bn,Cn,Dn] =th2ss(th);

$$
\begin{aligned}
& A_{n}=\left\lfloor\begin{array}{cc}
0.8283 & 0.4742 \\
-0.4622 & 0.5072
\end{array}\right], \quad B_{n}=\left\lfloor\begin{array}{l}
0.0074 \\
0.0162
\end{array}\right\rfloor \\
& C_{n}=[14.7560-1.7484], D_{n}=[1.5961 \mathrm{e}-004]
\end{aligned}
$$

As matrizes do sistema linear obtidas pelo método MOESP são dadas pelo seguinte algoritmo:

$[\mathrm{S}, \mathrm{R}]=$ dordpo $($ ue $, \mathrm{ze}, 30)$

$[\mathrm{Am}, \mathrm{Cm}]=\mathrm{dmodpo}(\mathrm{R}, 2)$

$[\mathrm{Bm}, \mathrm{Dm}]=\mathrm{dac} 2 \mathrm{bd}(\mathrm{Am}, \mathrm{Cm}, \mathrm{ue}, \mathrm{ze})$

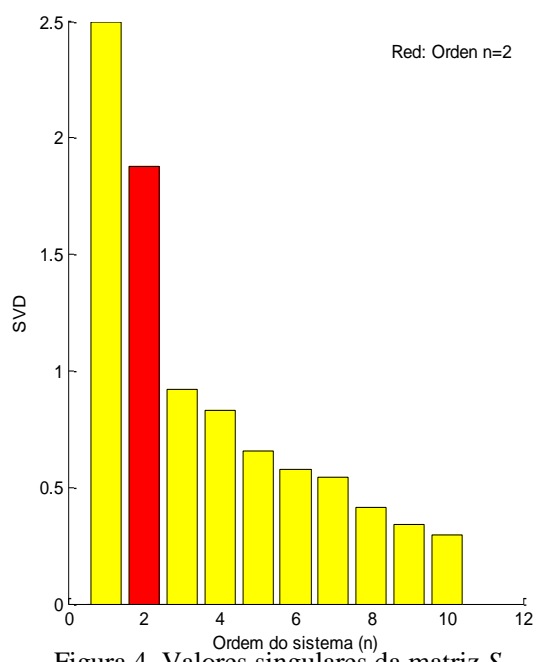

Figura 4. Valores singulares da matriz $S$

$$
\begin{aligned}
& A_{m}=\left\lfloor\begin{array}{cc}
0.7675 & 0.6214 \\
-0.3266 & 0.5883
\end{array}\right], \quad B_{m}=\left\lfloor\begin{array}{l}
-0.3136 \\
-0.1762
\end{array}\right] \\
& C_{m}=\left[\begin{array}{ll}
-0.5529 & 0.5149
\end{array}\right], D_{m}=[1.1908 \mathrm{e}-005]
\end{aligned}
$$

A estimativa da saída linear é dada por:

$\tilde{y}_{m}=\mathrm{dl} \operatorname{sim}(\mathrm{Am}, \mathrm{Bm}, \mathrm{Cm}, \mathrm{Dm}, \mathrm{u}(1: 1500,:))$;

$\tilde{y}_{n}=\operatorname{dl} \operatorname{sim}(\mathrm{An}, \mathrm{Bn}, \mathrm{Cn}, \mathrm{Dn}, \mathrm{u}(1: 1500,:))$;

para os casos MOESP e N4SID, respectivamente. O passo seguinte é estimar a função não linear $F(*)$, usando a saída estimada do sistema linear e a saída $z_{k}$ do modelo de Wiener. Esta estimativa é dada pelos polinômios de Chebyshev, para $n=13$. A estimativa de $C_{n}$ (e $C_{m}$ ), coeficientes da função não linear usando a saída $z_{k}$ e entrada $\tilde{y}_{n}$ (e entrada $\left.\tilde{y}_{m}\right)$ é:

$$
\begin{aligned}
& \mathrm{Cn}=\left[\begin{array}{lllll}
-0.0261 & 0.6269 & 0.0513 & -0.1922 & -0.0378
\end{array}\right. \\
& \begin{array}{lllll}
0.0975 & 0.0364 & -0.0402 & -0.0233 & 0.0419
\end{array} \\
& \left.\begin{array}{llll}
0.0212 & 0.4628 & 1.2214
\end{array}\right]
\end{aligned}
$$

$$
\begin{aligned}
& \mathrm{Cm}=\left[\begin{array}{lllll}
-0.0245 & 0.6247 & 0.0468 & -0.1954 & -0.0358
\end{array}\right. \\
& \begin{array}{llllll}
0.0988 & 0.0335 & -0.0407 & -0.0220 & 0.0464
\end{array} \\
& \left.\begin{array}{lll}
0.0211 & 0.4617 & 1.2350
\end{array}\right]
\end{aligned}
$$

Para se avaliar a qualidade do modelo, aplicam-se indicadores de desempenho, dentre os quais a média da variância relativa (MVAF), definida por:

$$
\operatorname{MVAF}(\%)=\frac{1}{l} \sum_{i=1}^{N}\left(1-\frac{\operatorname{var}(z-\hat{z})}{\operatorname{var}(z)}\right) \cdot 100
$$

onde $z$ é a saída real e $\hat{z}$ a saída estimada pelo modelo obtido. O índice MVAF é usado pelo SI (System Identification) Toolbox do Matlab. Este índice de desempenho é empregado para avaliar a qualidade do modelo produzido por cada algoritmo, como mostra a Tabela 1 . 
Tabela 1. Resultados numéricos do desempenho dos algoritmos.

\begin{tabular}{|l|l|l|}
\hline \multicolumn{1}{|c|}{ Algoritmos } & \multicolumn{1}{c|}{$\begin{array}{c}\text { MVAF auto- } \\
\text { validação (\%) }\end{array}$} & $\begin{array}{c}\text { MVAF validação } \\
\text { cruzada (\%) }\end{array}$ \\
\hline MOESP & 99.6716 & 99.6416 \\
\hline N4SID & 99.4703 & 99.4137 \\
\hline
\end{tabular}

Através dos dados apresentados na Tabela1, percebese que o modelo MOESP superou o modelo N4SID na autovalidação e na validação cruzada, motivo pelo qual se utiliza este modelo para identificar o sistema de Wiener.

A relação entre a saída estimada linear e a não linear $F(y)$ é mostrada na Figura 5. A linha contínua azul representa o polinômio de Chebyshev para $n=13$.

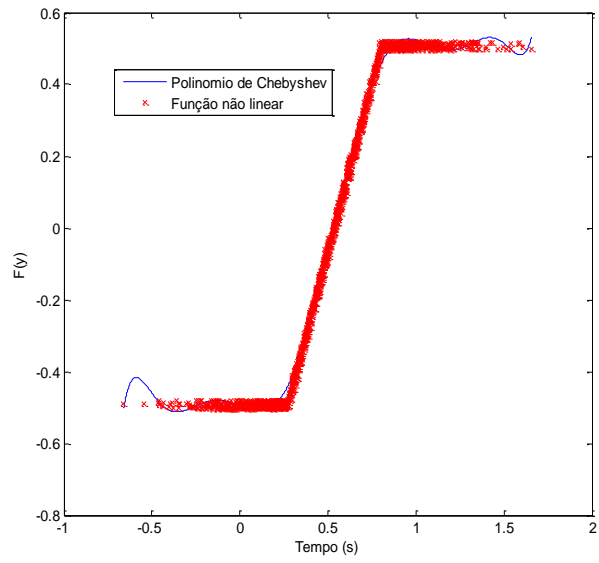

Figura 5. Comparação da função não linear real versus a estimada para dados sem ruído.

A Figura 6 mostra a saída do processo real (linha contínua) e aquelas geradas pelo polinômio de Chebyshev usando como entrada a saída do modelo MOESP (linha tracejada). Observa-se que o modelo identificado reproduz muito bem as principais características do processo. Foram consideradas condições iniciais nulas.

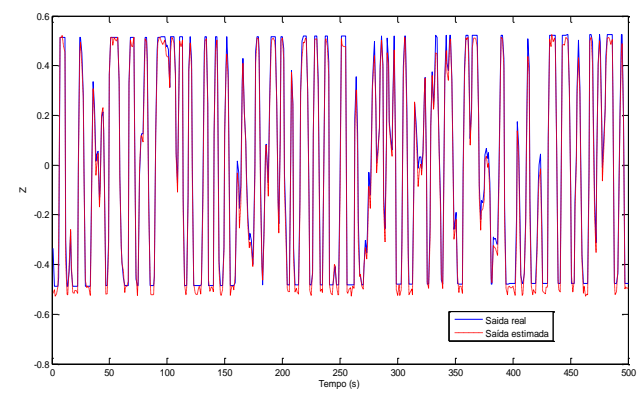

Figura 6. Saída real do processo e saída simulada usando o polinômio de Chebyshev.

A coleta de dados do processo real está sempre afetada por ruído. Então se agregou $3 \%$ de ruído aos dados de entrada e saída:

$\mathrm{ur}=\mathrm{u}+0.03 * \operatorname{std}(\mathrm{u}) * \operatorname{randn}(\operatorname{size}(\mathrm{u}))$;

$\mathrm{y}=\mathrm{dl} \operatorname{sim}($ num,den,ur);

$\mathrm{zr}=\mathrm{z}+0.03 * \operatorname{std}(\mathrm{z}) * \operatorname{randn}(\operatorname{size}(\mathrm{z}))$;

Para este caso, a ordem do sistema linear é $n=2$ e a ordem do polinômio de Chebyshev é $n=10$. O resultado da simulação é mostrado na Tabela 2 e a relação entre a saída estimada linear e a não linear $F(y)$ é mostrada na Figura 7. A linha contínua azul representa o polinômio de Chebyshev.

Tabela 2. Resultados numéricos dos algoritmos com 3\% de ruído.

\begin{tabular}{|l|l|l|}
\hline \multicolumn{1}{|c|}{ Algoritmos } & \multicolumn{1}{c|}{$\begin{array}{c}\text { MVAF auto- } \\
\text { validação (\%) }\end{array}$} & $\begin{array}{c}\text { MVAF validação } \\
\text { cruzada (\%) }\end{array}$ \\
\hline MOESP & 99.4344 & 99.3653 \\
\hline N4SID & 99.3426 & 99.3188 \\
\hline
\end{tabular}

Analisando-se os valores da Tabela2, o modelo MOESP teve melhor desempenho.

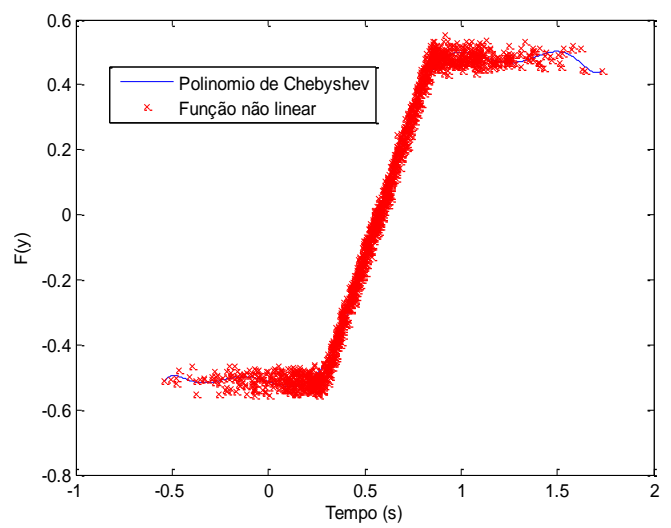

Figura 7. Comparação da função não linear real versus a estimada para dados com $3 \%$ de ruído.

\section{Conclusão}

Neste trabalho foi mostrada uma metodologia para a identificação de um sistema tipo Wiener usando métodos por subespaços e polinômios de Chebyshev. Um exemplo simulado do tipo Wiener é apresentado usando essa metodologia. Para este exemplo simulado, o método MOESP teve melhor desempenho na identificação do bloco linear em relação ao modelo N4SID, de acordo com o critério MVAF. A partir das Tabelas 1 e 2 e das Figuras 5 e 6 pode-se visualizar que os polinômios de Chebyshev aproximam muito bem a função não linear onde os dados são avaliados.

\section{Agradecimentos}

Agradecemos a FAPERN pelo apoio financeiro na realização deste trabalho.

\section{Referências Bibliográficas}

Biagiola, S; Figueroa, L (2011). Identification of uncertain MIMO Wiener and Hammerstein models, Computers \& Chemical Engineering, vol. 35 , no. 11 , pp. 1-9.

Billings, S (1980). Identification of nonlinear systems a survey. In Proc. IEEE, Part D, pp. 272-285.

Bloemen, H; Chou, C; Van den Boom, T; Verdult, V. Verhaegen, M; Backx, T. (2001). Wiener model 
identification and predictive control for dual composition control of a distillation column Journal of Process Control, vol. 11, pp. 601620. DOI: $10.1016 / \mathrm{S} 0959-1524(00) 00056-1$

Borjas, S; Garcia, C (2012). Identificação Determinística por Subespaços. TEMA. Tendências em Matemática Aplicada e Computacional, vol. 13, pp. 207-218. DOI: 10.5540/tema.2012.013.03.0207

Goethals, K; Pelckmans, J. A; Suykens, and De Moor, B (2004). Identification of MIMO Hammerstein models using least squares support vector machines. ESAT-SISTA, Leuven, Belgium, Tech. Rep. PP. 04-45.

Greblicki, W. and Pawlak, M. (1991). Nonparametric identification of a cascade nonlinear time series system. Signal Processing, vol. 22, pp.61-75. DOI: 10.1016/0165-1684(91)90029-I

Lovera, M; Gustafsson, T. and Verhaegen, M (2000). Recursive subspace identification of linear and non-liearWiener state space models. Automatica, vol. 36, pp. 1639-1650. DOI: 10.1016/S0005-1098(00)00103-5

Namatame, A; Ueda, N (1992). Pattern classification with Chebyshev neural networks. Int. J. Neural Networks, vol. 3, pp. 23-31.

Patra, J.C.; Kot, A.C (2002). Nonlinear dynamic system identification using chebyshev functional link artificial neural networks. IEEE Trans. Systems, Man \& Cybernetics, Part-B, vo1.32, no.4, pp.505-511. DOI: 10.1109/TSMCB.2002.1018769

Pearson, R.; Pottmann, M (2000). Gray-box identification of block-oriented nonlinear models Journal of Process Control, vol. 10, pp. 301-315. DOI: 10.1016/S0959-1524(99)00055-4

Purwar, S; Kar, I.N.; Jha, A.N.(2003). On-line system identification using Chebyshev neural networks, in: Proceedings of IEEE TENCON-2003 Conference, Bangalore, India.

Rivlin, T. J (1990). Chebyshev Polynomials Form Approximation Theory to Algebra and Number Theory, 2nd ed. New York: Wiley, ch.3, pp. 155-191.

Saha, P.; Krishnan, S.; Rao, V.; Patwardhan, S. (2004). Modeling and predictive control of MIMO nonlinear systems using WienerLaguerre models. Chemical Engineering Communication, vol. 191, pp. 1083-1119. DOI: 10.1080/00986440490276452

DOI: $10.1080 / 0098644049276452$

Shingu, T; Takata, H. and Hachino, T. (1997). An Identification Method of Discrete Nonlinear Systems via Chebyshev Interpolation and its Error Bound, NOLTA'97, vol. 01, No. 2, pp. 11251128.

Van Overschee, P. and De Moor, B. (1996). Subspace Identification for Linear Systems. Kluwer Academic Pub. DOI:10.1007/978-1-4613-0465-4

Verhaegen, M. (1994). Identification of the deterministic of MIMO state space models give in innovations form input-output data, In the Special Issue on Statistical Signal Processing and Contro
Volterra, V. (1959). Theory of Functional end of Integral and Integro-Differential Equations. New York, Dover.

Westwick, D. and Verhaegen, M. (1996). Identifying MIMO Wiener systems using subspace model identification methods. Signal Processing, vol. 52, pp. 235-258. DOI: 10.1016/0165-1684(96)00056-4

Wigren, T (1994). Convergence analysis of recursive identification algorithms based on the nonlinear Wiener model. IEEE Trans. AC, vol. 39, pp. 2191-2206. DOI: 10.1109/9.333765

Zhu, Y (1999). Distillation column identification for control using Wiener model. In 1999 American Control Conference. DOI: 10.1109/ACC.1999.782408 